\title{
Performance Evaluation of Different Classifier for Eye State Prediction Using EEG Signal
}

\author{
Mridu Sahu, N. K. Nagwani, Shrish Verma, and Saransh Shirke
}

\begin{abstract}
Electroencephalography (EEG) measure human brain activity for different applications. In this paper performance of Instance Based classifier is compared with well known classifier, such as AdaBoost, J48, IB1, etc. As the experimental result shows that the Random forest and Instance Based Classifier like IB1\& IBK are performing well as compared to other classifiers for predicting eye state (open or closed) using EEG signals from electrode data.
\end{abstract}

Index Terms-Electroencephalography (EEG), emotive devices, instance based classifier.

\section{INTRODUCTION}

Classification is the task of data mining to make a group of data that are similar to each other placed in similar class. The Instance Based classifier is a machine learning method that classifies new unseen data by comparing them to those all ready seen in memory [1]. In machine learning and statistics, classification is used to identify the class or categories based on the data. On the basis of a training set of data containing instances class is described. In the terminology of machine learning [2], classification is considered an instance of supervised learning, where training set classify the instances on basis of trained set or by any other method.

There are various types of Instance Based classifier such as KNN, IBL (Instance Based learning IB1, IB2, IBK, K-star). IBL algorithms are mostly used in domain specific system and industrial applications like ALFA [3]. IBL algorithms are derived from the nearest neighbor (KNN) pattern classifier [4] but the KNN requires more space and time as compared to IBL algorithms shown in different literatures. Instance Based learner stores all the training examples when seeing a new instance it look at most similar store instance and make prediction on the basis of those instances. The classical example of IBL is $\mathrm{K}$ nearest neighbor. It finds $\mathrm{K}$ most similar instances and gives most frequent class or means target value as prediction the proposal work using Table I, data for predicting eye state. The advantages of IBL are fast learning

Manuscript received March 2, 2015; revised June 3, 2015. This research is supported by the National Institute of Technology, Raipur, India and many thanks to WEKA machine learning group as well as Rosler and Suendermann from Baden-Wuerttenberg Cooperative State University (DHBW), Stuttgart, Germany for providing EEG Corpus.

Mridu Sahu is with the Department of IT, NIT, Raipur, India (e-mail: mrisahu.it@nitrr.ac.in).

Saransh Shirke is with the Department of Electrical Engineering, NIT Raipur, India (e-mail: saransh.shirke@gmail.com).

K. Nagwani is with the Department of CSE, NIT, Raipur, India (e-mail: nknagwani.cs@nitrr.ac.in).

Shrish Verma is with the Department of E\&TC, NIT, Raipur, India (e-mail: shrishverma@nitrr.ac.in). (just storing the instances) and no information is last all the instances are kept. IBL is a lazy learner. Lazy learning is kind of novel machine learning method based on statistical learning theory which based on memory learning strategy [5].

A common data mining task is the estimation of similarity among instances. A similarity measure measures relationship between pair of instances [5]. The IBL is uses the similarity functions as:

$$
x_{i}, y_{i}=\left(x_{i}-y_{i}\right)^{2}
$$

The instances is described by attributes, it is defined by numeric value attributes and $f\left(x_{i}, y_{i}\right)=\left(x_{i} \neq y_{i}\right)$ for Boolean and symbolic attributes values [6]. Various IBL algorithms like IB1, IB2, IBK, and K-star are well known Instance Based Classifier.

The next step for IBL is a feature selection and it is also known as variable selection, attribute selection or variable subset selection. It is the process of selecting a subset of relevant feature for model construction. Feature selection techniques are a subset of feature extraction. Feature extraction creates new feature from the original feature whereas feature selection is a process of selecting subset of the features which are used to classify the class on basis of instances of data found in dataset.

In proposed paper, classification is based on all feature space extracted from electrode position. Further extension of proposed work is to regenerate new features from this electrode time series data then find optimal feature selection for better classification. In experiment various classifier algorithms are used and classification accuracy is compared with different parameters .Here classification algorithms like Tree based, Naivebayes, Rule based, and Meta classifier are used for classification of eye state weather eye is closed or open.

\section{ELECTROENCEPHALOGRAPHYSYSTEM (EEG)}

The EEG is a kind of machine which produces a graph measurement of brain waves known as Electroencephalography [7]. It is most often used to diagnose epilepsy, sleep disorders, coma, encephalopathies, and brain death which cause abnormalities in EEG readings [8]. It is first-line method of diagnosis for tumors, stroke and other focal brain disorders [9]. There are various spatial resolution of EEG systems like 10/20,10/10,10/5 systems as relative had surface based positioning system. The international 10/20 system a standard system for electrode positioning with 21 electrodes extended to higher density electrode such as 10/10 and 10/5 systems allowing more than 300 electrode positions [10]. Emotive headset is a device which gives the value at 
each instance shown in Fig. 1.

The system involves hooking up several pairs of electrodes on patient head (16 electrode named as F7, F3, F4, FC6, T8, P8,O2,CMS as eye open state and AF3, AF4, FC5, F8, T7, P7, O1 DRL as eye closed state) shown in Fig. 2. These electrodes are disc that conducts electrical activity and captures it from brain and convert it out through a wire to a machine that amplifies the signal [11]. Our brain works every time even sleeping, so the electrical current even passes when we are sleeping. Brain waves a usually slowest during sleep. The proposed work is applicable for finding eye state classification in the area of infant sleep walking state identification [12], driving drowsiness detection [13], epileptic seizure detection [14], classification of bipolar mood disorder (BMD) and attention deficit hyperactivity disorder (ADHD) patients [15], stress features identification [16], human eye blinking detection [17].

\section{DESCRIPTION OF DATA SET}

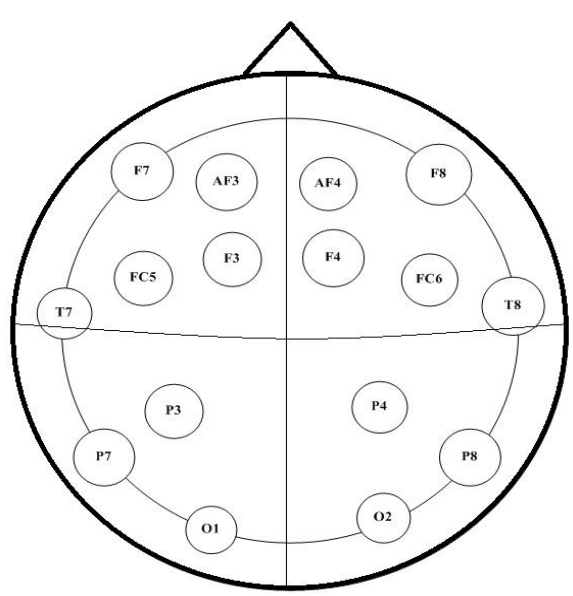

Fig. 1. EEG 10/20 machine system.

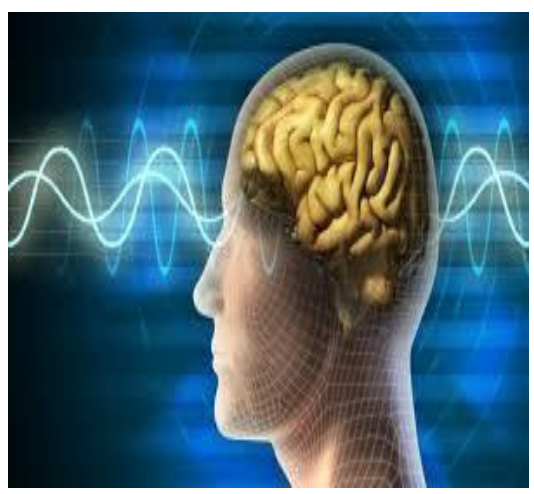

Fig. 2. EEG signal waves

The Corpus consists of 14980 instances with 15 features each (14 features representing the values of Electrodes and 1 eye state open or close). Outliers in statistics observation point which is different than other observation points [18]. It shows experimental error due to variability in measurement of dataset so removal of outliers is required [18]. After Outlier selection the corpus consists of 14977 instances, three instances with the numbers 899, 10387, and 11510 having obvious errors i.e. outliers. The instances are stored in the corpus and ordered as to analyze temporal dependency $8255(55.12 \%)$ instances of the corpus corresponds to the eye open and 6722(44.88\%) instances to the eye closed. The corpus is taken from the link [19], and it was donated by Rosler's and Suendermann from Baden-Wuerttemberg Cooperative State University (DHBW), Stuttgart, Germany [19]. The output of the corpus, " 1 " indicates the eye-closed and " 0 " the eye-open. Table I shows the min, mean, max values of Eye closed and Eye open ranges of the 14 sensors in the corpus [19]. The output of the corpus, "1" indicates the eye-closed and " 0 " the eye-open.

TABLE I: RANGES AND MEANS OF THE SENSOR VALuES FOR THE EyE STATES

\begin{tabular}{|l|l|l|l|l|r|l|}
\hline \multirow{2}{*}{ Eye State } & \multicolumn{6}{|l}{ Close } \\
\cline { 2 - 7 } & Min & Mean & Max & Min & Mean & Max \\
\hline AF3 & 4198 & 4305 & 4445 & 1030 & 4297 & 4504 \\
\hline F7 & 3905 & 4005 & 4138 & 3924 & 4013 & 7804 \\
\hline F3 & 4212 & 4265 & 4367 & 4197 & 4263 & 5762 \\
\hline FC5 & 4058 & 4121 & 4214 & 2453 & 4123 & 4250 \\
\hline T7 & 4309 & 4341 & 4435 & 2089 & 4341 & 4463 \\
\hline P7 & 4574 & 4618 & 4708 & 2768 & 4620 & 4756 \\
\hline O1 & 4026 & 4073 & 4167 & 3581 & 4071 & 4178 \\
\hline O2 & 4567 & 4616 & 4695 & 4567 & 4615 & 7264 \\
\hline P8 & 4147 & 4202 & 4287 & 4152 & 4200 & 4586 \\
\hline T8 & 4174 & 4233 & 4323 & 4152 & 4229 & 6674 \\
\hline FC6 & 4130 & 4204 & 4319 & 4100 & 4200 & 5170 \\
\hline F4 & 4225 & 4281 & 4368 & 4201 & 4277 & 7002 \\
\hline F8 & 4510 & 4610 & 4811 & 86 & 4601 & 4833 \\
\hline AF4 & 4246 & 4367 & 4552 & 1366 & 4356 & 4573 \\
\hline
\end{tabular}

\section{LITERATURE REVIEW ON DIFFERENT CLASSIFIERS}

An algorithm that implements classification, especially in a concrete implementation, is known as a classifier. The term "classifier" sometimes also refers to the mathematical function, implemented by a classification algorithm, which maps input data to a category. Description of different classifier used for experiment in this work.

J48: J48 is a classifier using divide and conquer strategy for decision making [20]. Divide and conquer strategy is solved by recursive procedure or recursive tree procedure based on this; it will create desiccation tree for classification of eye state whether it is open or closed. A decision tree helps decision support system and it uses tree like structure. It is used to learn a classification function it requires dependant variable and independent variable for classification [21]. Decision tree have many advantages over other classification algorithms because it is having ability to work with variety of input data types (nominal scale, numeric scale and textual data) this classifier is tested using weka. The performance of this classifier is $83.2415 \%$ for current corpus.

Decision Stump: It is a one type of machine learning model. It is the simplest form of weak classifier because the decision tree with a single node only for decision making, so that this classifier is known as decision stump. The advantage of this classifier is its simplicity. The proposed article used decision stump to compare the classification accuracy with others and found that it is not good classifier for eye state classification [22]. The eye state classification accuracy is found $59.4058 \%$.

Random Forest: Random forests (RF) are a classification algorithm that uses an ensemble method [23]. It is also using tree like structure for decision making and performing aggregates on it. Randomness is completed with two steps 
first is bootstrap sample construct for each tree than tree split for best branch selection [24]. The article founds the accuracy of eye state classification is $89.272 \%$. Its performance is better than Naivebayes, ada boost, baging, etc. and lower than instance based classifier.

Random Tree: Leo Breiman and Adele Cutler introduced random tree classification algorithm. The beauty of this algorithm is that it works with both regression and classification problems. Random tree doesn't require accuracy estimator [25]. Random decision tree algorithm built multiple decision trees randomly. Eye state classification accuracy computed by this algorithm is $81.2886 \%$.

REP tree: Rep tree is also known as regression tree representative. It uses linked based feature, it can be used to model functions and predicted value of each point. It also creates multiple trees in different iterations [26]. Eye state classification accuracy computed by this algorithm is $82.5572 \%$.

Naivebayes: This method is oldest method for classification it is using probability theory for classification process. It is a supervised type of learning algorithm and literature said that it is extremely fast as compare to sophisticated methods. Article founds the accuracy of eye state classification is $62.861 \%$ and it is very less as compare to most of the classifier used in classification.

AdaBoost: It is an idea to combine relatively weak and inaccurate rules. The initial requirement of ada boost is first enough training samples second good fit to training samples and third is it should be simple [27]. The accuracy computed by this algorithm is $68.3692 \%$.

Bagging: It is a procedure of combining different decision making samples and learning algorithms with one frame. The training data is divided into " $n$ " number of samples and giving different learning algorithms to each sample than the new classification comes after combining all learning rules and classifier to create a new combine classifier is known as bagging because in its bag " $n$ " number classifier. Eye state classification accuracy computed by this algorithm is $86.9304 \%$.

Classification via regression: It gives better understanding of classification outcomes. It is a supervised type of learning. Eye state classification accuracy is found $84.3933 \%$.

Decision table: Decision table checks knowledge base for completeness and consistency. It creates a huge table for decision from rule based knowledge and splits the table into sub tables with similar logic and checks consistency and completeness [28]. Classification founds by Decision table classifier is $72.8092 \%$.

TABLE II: VARIOUS MEASURE OF CLASSIFIER

\begin{tabular}{|c|c|c|c|c|c|c|c|c|}
\hline Classifiers & TP Rate & FP Rate & Precision & Recall & F-Measure & ROC Area & Accuracy & Error Rate \\
\hline IB1 & 0.945 & 0.056 & 0.945 & 0.945 & 0.945 & 0.945 & $94.5087 \%$ & $5.4916 \%$ \\
\hline $\mathrm{IBK}=2$ & 0.927 & 0.085 & 0.931 & 0.927 & 0.927 & 0.965 & $92.7224 \%$ & $72776 \%$ \\
\hline $\mathrm{IBK}=3$ & 0.934 & 0.067 & 0.934 & 0.934 & 0.934 & 0.974 & $93.4235 \%$ & $6.5765 \%$ \\
\hline $\mathrm{IBK}=4$ & 0.925 & 0.084 & 0.926 & 0.925 & 0.924 & 0.976 & $92.472 \%$ & $7.528 \%$ \\
\hline $\mathrm{IBK}=5$ & 0.927 & 0.074 & 0.927 & 0.927 & 0.927 & 0.977 & $92.7391 \%$ & $7.2609 \%$ \\
\hline $\mathrm{J} 48$ & 0.832 & 0.174 & 0.832 & 0.832 & 0.832 & 0.846 & $83.2415 \%$ & $16.7585 \%$ \\
\hline Decision Stump & 0.594 & 0.488 & 0.634 & 0.594 & 0.516 & 0.553 & $59.4058 \%$ & $40.5942 \%$ \\
\hline Random Forest & 0.892 & 0.120 & 0.895 & 0.892 & 0.891 & 0.958 & $89.272 \%$ & $10.7828 \%$ \\
\hline Random Tree & 0.813 & 0.191 & 0.813 & 0.813 & 0.813 & 0.811 & $81.2886 \%$ & $18.7114 \%$ \\
\hline REP tree & 0.826 & 0.184 & 0.826 & 0.826 & 0.825 & 0.864 & $82.5572 \%$ & $17.4428 \%$ \\
\hline AdaBoost & 0.684 & 0.343 & 0.684 & 0.684 & 0.678 & 0.723 & $68.3692 \%$ & $31.6308 \%$ \\
\hline Bagging & 0.869 & 0.140 & 0.870 & 0.869 & 0.869 & 0.943 & $86.9304 \%$ & $13.0696 \%$ \\
\hline Classification Via Regression & 0.844 & 0.164 & 0.844 & $0 . .844$ & 0.844 & 0.918 & $84.3933 \%$ & $15.6067 \%$ \\
\hline Decision table & 0.728 & 0.296 & 0.730 & 0.728 & 0.724 & 0.802 & $72.8092 \%$ & $27.1908 \%$ \\
\hline SMO & 0.652 & 0.398 & 0.664 & 0.652 & 0.629 & 0.627 & $65.1811 \%$ & $34.8189 \%$ \\
\hline
\end{tabular}

SMO: Sequential minimal optimization (SMO) is an algorithm used to solve the quadratic programming problem which arises during training of support vector machine [29]. It is divided into sub problems and solved analytically. Accuracy founds by this classifier is very less compare to other classifier i.e. $65.1811 \%$.

This uses the instance based classifier $\left(\mathrm{K}^{*}\right)$ based on statistic of data and nature of data spread over the corpus found it is best among other classifier the result of this present in literature is best in Instance based classifier compare to other classifier [30]. Table II shows the result of different classifiers which includes TP Rate, FP Rate, Precision, Recall, F-measure, ROC curve, Accuracy and Error Rate.

\section{A. Experiment}

In proposed work the EEG dataset for experiment is taken and the process diagram is shown in Fig. 3. After data extraction outlier detection is performed for purifying the extracted data from different electrode positions. After this feature estimation is completed, only 14 features are selected from all features.

Then it performs feature classification based on different types of classifier such as Tree based, Meta based, Bayesian based, Function based etc. The various statically measures for classifier is evaluated and different measure like TP Rate, FP Rate, Precision, Recall, F-measure, ROC area are calculated 
and listed it in Table II .Accuracy of Instance Based classifier is compared with other classifier and it is shown in Fig. 4.

TPR (True Positive Rate): It is also known as sensitivity which measures the actual positive classified instances in binary classification.

$$
T P R=T P /(T P+F N)
$$

where :

$T P$ is True positive,

$F N$ is False Negative.

FPR (False Positive Rate): It is also known as false alarm ratio. It measures the expectancy of false positive ratio of instances classified.

$$
F P R=F P /(F P+T N)
$$

where:

$F P$ is false positive

$T N$ is True Negative.

Precision: The proportion of predicted positive which are actual positive. It is the fraction of retrieved instances that are relevant in recognition with binary classification. It is also known as positive predicted value.

$$
\text { Precision }=T P /(T P+F P)
$$

where:

$T P$ is True positive

$F P$ is False Positive.

Recall: The proportion of actual positive which are predicted positive. It is the fractions of relevant instances that are retrieved instances which are recognize the class in classification.

$$
\text { Recall }=T P /(T P+F N)
$$

where

$T P$ is True positive

$F N$ is False Negative.

F-measure: It is harmonic mean between Precision and Recall and also known as F-score. It considers both the precision and the recall of the test to compute the score: precision is the number of correct positive results divided by the number of all positive results, and recall is the number of correct positive results divided by the number of positive results that should have been returned. The F-score can be interpreted as a weighted average of the precision and recall [31].

F-measure $=2($ Precision $\times$ Recall $) /($ Precision + Recall $)[32]$

ROC curve (Receiver Operating Characteristic curve): In statistics, a receiver operating characteristic (ROC), or ROC curve, is a graphical plot that illustrates the performance of a binary classifier system as its discrimination threshold is varied. The curve is created by plotting the true positive rate against the false positive rate at various threshold settings. It is a $2 \mathrm{D}$ curve parameterized by one parameter of the classification algorithm. In a ROC curve the TP rate (sensitivity) is plotted in function of FP rate for different cut-off points of parameter [33]. The ROC is also known as a relative operating characteristic curve, because it is a comparison of two operating characteristics (TPR and FPR) as the criterion changes [33].

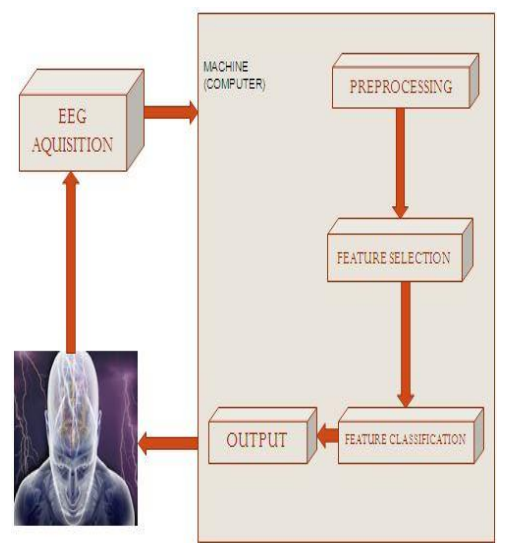

Fig. 3. Execution of proposed work.

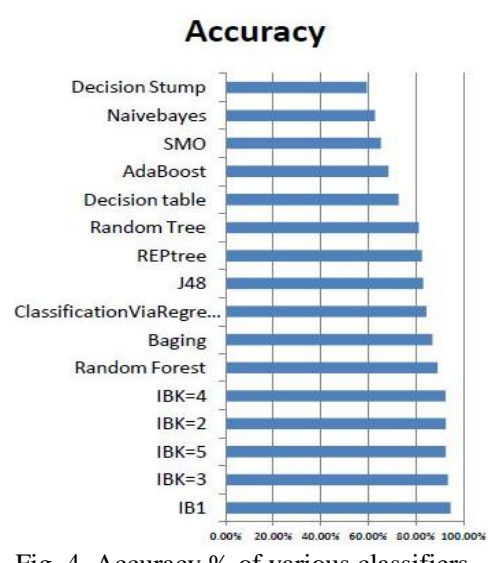

Fig. 4. Accuracy $\%$ of various classifiers.

The proposed article experiment or method for classifying the binary class data of eye detection whether eye is close or open with the help of different classifier and the steps of the process is given below stepwise. Basically the classification has been done by the "weka" machine learning tool and the results has been shown in table and by the accuracy rate the best classifier for the eye state classification is declared in this proposed article.

Step wise description for classifying eye state (open or close)

Step 1: Take input as Corpus.

Step 2: Data preprocess as Outliers present in corpus and removal from corpus.

Step 3: Create three sets training, testing, validation.

Step 4: 15 features had been selected 14 electrodes value and 1 class as eye detection (open or close).

Step 5: Classify the train data with tested data by different classifiers
1) IB 1
2) $\mathrm{IBK}=2$
3) $\mathrm{IBK}=3$
4) $\mathrm{IBK}=4$
5) $\mathrm{IBK}=5$
6) $\mathrm{J} 48$
7) Decision Stump
8) Random Forest
9) Random Tree
10) REPtree
11) Naivebayes
12) AdaBoost 
13) Bagging

14) ClassificationViaRegression

15) Decision table

16) $\mathrm{SMO}$

Step 6: Store the Results of different classifier.

Step 7: Based on Accuracy rate best classifier is declared for eye state detection (open or close).

\section{CONCLUSION}

In this paper, IB classifier accuracy is compared with different classifier. KNN ( $K$-nearest neighbor) is also a instance based classifier but the space requirement is very large for this classifier.IBK is a $\mathrm{K}$ instance based classifier used in this EEG corpus, the value of $K=3$ gives better result among value of 2, 3, 4 and 5 and it shows it is best classifier among trees based, lazy classifier, Rules based, meta based, bayes based, functions based classifier.IBL accuracy is $94.5 \%$. So the instance based classifier is better choice to predict whether the Eye State is open or closed.

\section{ACKNOWLEDGEMENT}

This research is supported by the National Institute of Technology, Raipur, India and many thanks to WEKA machine learning group as well as Rosler and Suendermann from Baden-Wuerttenberg cooperative state university (DHBW), Stuttgart, Germany for providing EEG corpus.

\section{REFERENCES}

[1] D. W. Aha, D. Kibler, and M. K. Albert, Machine Learning, Springer, 1991.

[2] A. Ethem. Introduction to Machine Learning, MIT press, 2014.

[3] K. Jabbour et al., "ALFA: Automated load forecasting assistant," presented at the IEEE Power Engineering Society Summer Meeting, San Francisco, CA. 1987.

[4] T. M. Cover and P. E. Hart, "Nearest neighbor pattern classification," Institute of Electrical and Electronics Engineers Transactions on Information Theory, vol. 13, pp. 21-27, 1967.

[5] C. Carmelo, P. Montalto, M. Aliotta, A. Cannata, and A. Pulvirenti. "Similarity measures and dimensionality reduction techniques for time Series data mining," Advances in Data Mining Knowledge Discovery and Applications, 2012.

[6] D. W. Aha and D. Kibler, "Noise-tolerant instance-based learning algorithms," in Proc. the Eleventh International Joint Conference on Artificial Intelligence, pp. 794-799, Detroit, MI: Morgan Kaufmann, 1989.

[7] O. R"osler and D. Suendermann, "First step towards eye state prediction using EEG," in Proc. the International Conference on Applied Informatics for Health and Life Sciences (AIHLS '13), Istanbul, Turkey, 2013.

[8] F. Mohammad, G. Azemi, and B. Boashash, "EEG-based automatic epilepsy diagnosis using the instantaneous frequency with sub-band energies," in Proc. 2011 7th International Workshop on Systems, Signal Processing and their Applications (WOSSPA), pp. 187-190, IEEE, 2011.

[9] EEG.

[Online].

Available: http://www.nlm.nih.gov/medlineplus/ency/article/003931.htm

[10] T. Vernon et al., "The spatial location of EEG electrodes: Locating the best-fitting sphere relative to cortical anatomy," Electroencephalography and Clinical Neurophysiology, vol. 86, no. 1 1993, pp. 1-6.

[11] Emotiv EPOC works. [Online]. Available: http://electronics.howstuffworks.com/emotiv-epoc1.htm

[12] P. A. Est'evez, C. M. Held, C. A. Holzmann et al., "Polysomnographic pattern recognition for automated classification of sleep-waking states in infants," Medical and Biological Engineering and Computing, vol. 40, no. 1, pp. 105-113, 2002.
[13] M. V. M. Yeo, X. Li, K. Shen, and E. P. V. Wilder-Smith, "Can SVMbe used for automatic EEG detection of drowsiness during car driving?" Safety Science, vol. 47, no. 1, pp. 115-124, 2009.

[14] K. Polat and S.G"unes, "Classification of epileptiform EEG using a hybrid system based on decision tree classifier and fast Fourier transform," Applied Mathematics and Computation, vol. 187, no. 2, pp. 1017-1026, 2007.

[15] K. Sadatnezhad, R. Boostani, and A. Ghanizadeh, "Classification of BMD and ADHD patients using their EEG signals," Expert Systems with Applications, vol. 38, no. 3, pp. 1956-1963, 2011.

[16] N. Sulaiman, M. N. Taib, S. Lias, Z. H. Murat, S. A. M. Aris, and N. H. A. Hamid, "Novel methods for stress features identification using EEG signals," International Journal of Simulation: Systems, Science and Technology, vol. 12, no. 1,pp. 27-33, 2011.

[17] M. Grochowski and N. Jankowski, "Comparison of instance selection algorithms II," Results and Comments, pp. 580-585.

[18] E. Frank, "Procedures for detecting outlying observations in samples," Technometrics, vol. 11, no. 1, pp. 1-21, 1969.

[19] Corpus. [Online]. http://suendermann.com/corpus/EEG_Eyes.arff.gz

[20] H. Indriana, P. Erna, and M. A. Kristy, "Application of J48 and bagging for classification of vertebral column pathologies," in Proc. 2014 International Conference on Information Technology and Multimedia (ICIMU), IEEE, pp. 314-317, 2014.

[21] N. G. Sharma et al., "Decision tree analysis on j48 algorithm for data mining," International journal of Advanced Research in Computer Science and Software Engineering, vol. 3, no. 6, 2013.

[22] J. Xie, Y. Lu, Z. Lei, and X. Hui, "Boosting decision stumps to do pairwise classification," Electronics Letters, vol. 50, no. 12, pp. 866-868, 2014.

[23] L. Breiman, "Random forests," Machine Learning, vol. 45, pp. 5-32, 2001.

[24] Z.-P. Yi and J.-C. Pan, "Application of random forest to stellar spectral classification," in Proc. 2010 3rd International Congress on Image and Signal Processing (CISP), IEEE, vol. 7, pp. 3129-3132, 2010.

[25] S. K. Jayanthi and S. Sasikala, "Reptree classifier for identifying link spam in web search engines," Ictact Journal on Soft Computing, vol. 3, no. $2,2013$.

[26] Naive $\quad$ bayes. $\quad$ Anailable: http://scikit-learn.org/stable/modules/naive_bayes.html

[27] E. S. Robert, "Explaining adaboost," Empirical Inference, pp. 37-52, 2013.

[28] J. C. Brian and H. J. Steudel, "A decision-table-based processor for checking completeness and consistency in rule-based expert systems," International Journal of Man-Machine Studies, vol. 26, no. 5, pp. 633-648, 1987.

[29] P. John, "Sequential minimal optimization: A fast algorithm for training support vector machines," 1998.

[30] S. Mridu, N. K. Nagwani, S. Verma, and S. Shirke, "An incremental feature reordering (IFR) algorithm to classify eye state identification using EEG," Information Systems Design and Intelligent Applications, pp. 803-811, 2015.

[31] P. D. Martin, "Evaluation: From precision, recall and F-measure to ROC, informedness, markedness and correlation," 2011.

[32] Binary classification performances measure. [Online]. Available: http://www.damienfrancois.be/blog/files/modelperfcheatsheet.pdf

[33] A. S. John, Signal Detection Theory and ROC Analysis in Psychology and Diagnostics, Collected Papers, Psychology Press, 2014.

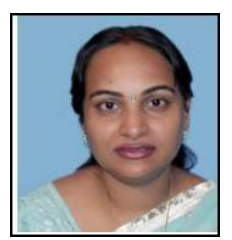

Mridu Sahu is an assistant professor in the Department of IT from NIT, Raipur and she is pursuing the PhD from NIT, Raipur as a part time candidate. She has 10 years teaching experience and her areas of interest are data mining, machine learning, analysis and design of algorithm. She published nine papers in national and international journals in area of data mining and machine learning.

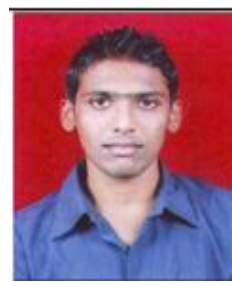

Saransh Shirke is pursuing MTech from the Department of Electrical Engineering from NIT Raipur, India. He completed BTech (IT) from JNTU, Hyderabad (AP) in 2011. His area of interest is data mining and machine learning. 